\section{A trick of the light \\ Discovering Dark-Bright and Anti-Dark-Bright Solitons}

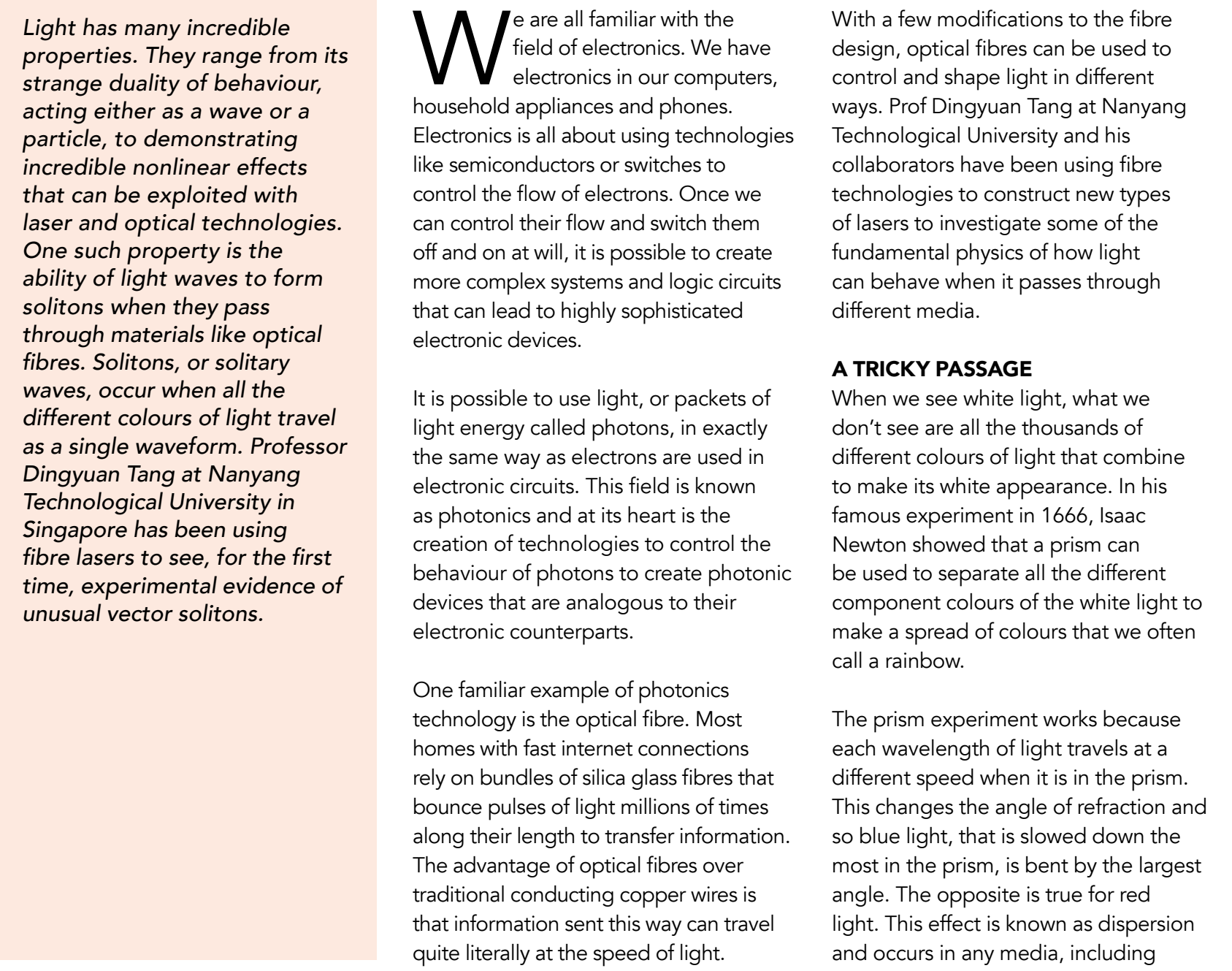

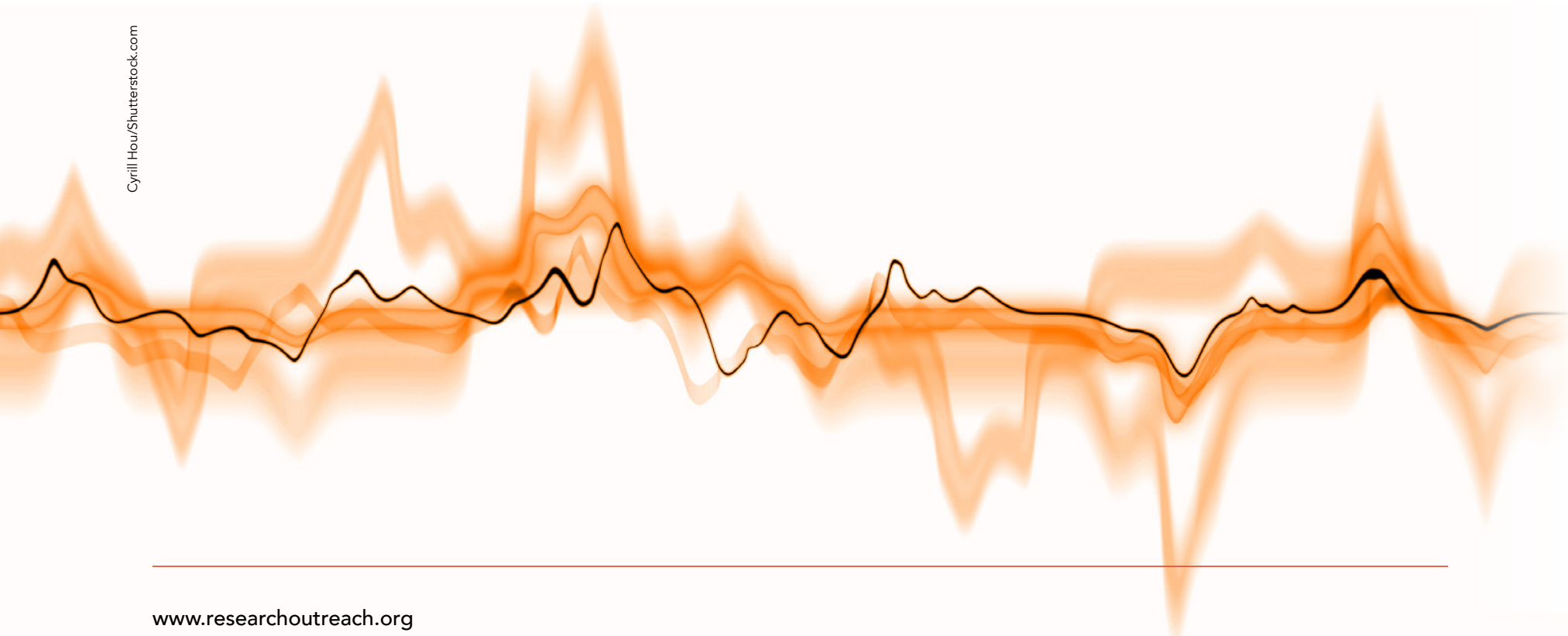

White Light

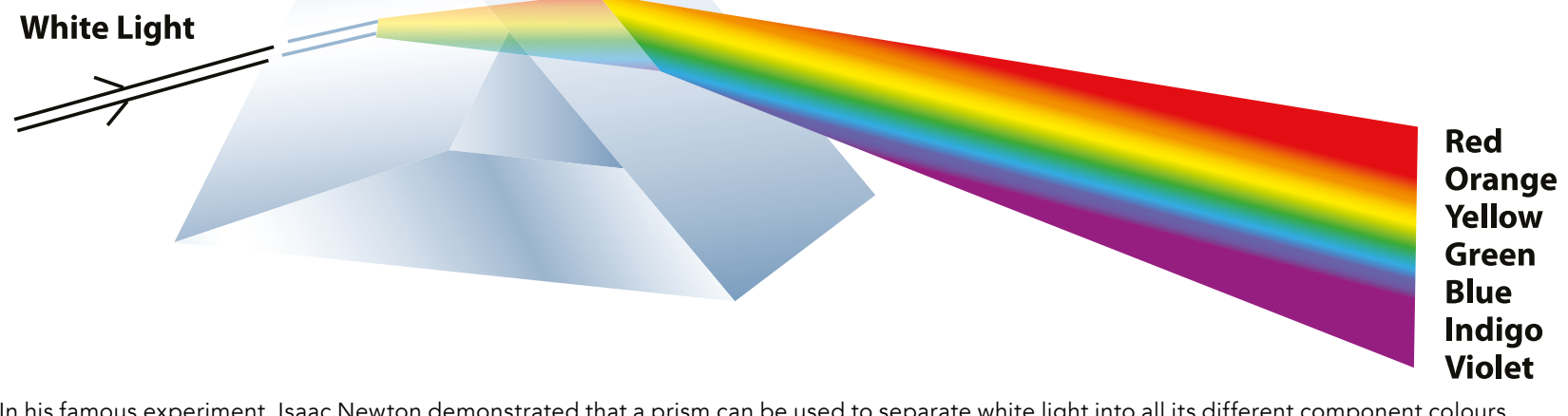

optical fibres. If we think of the intensity distribution of a single pulse of light entering a fibre, that might look like a brow, peaked $f$ distain, inere is a la light o will exit looking like a much broader, flatter hill.

Controlling the degree of dispersion caused by a fibre is one way of controlling the light pulse but there another effect to contend with, which is particularly important when intense light sources like lasers are used. This is the optical Kerr effect. Since light is an electromagnetic wave, whenever it enters a medium like a prism, the oscillating electric field of the incoming beam changes the refractive index of the medium. This, in turns, changes the amount of dispersion that the materia causes the light to undergo and is an example of a nonlinear

Prof Tang has been fascinated by the regime in which these dispersive nonlinear effects are perfectly balanced. Normally, as light passes through long fibres, the dispersive effects cause individual wavelengths to trave differently, changing the shape of the light pulse. Each of these wavelength will also experience a degree of self-phase modulation related to the strength of their electric field.

However, in a regime where the amoun of dispersion inherent to the material is compensated by the changes caused by the self-phase modulation, it is possible for the light pulse to pass through the
fibre completely unchanged in shape
It is possible to use light, or packets of light energy called photons, in exactly the same way as electrons are used in electronic circuits.

and form - this is known as a soliton Other types of waves, such as tidal waves on rivers, can also form solitons speeds.

LASER PULSE SHAPING

Prof Tang's interest has been in finding experimental methods to observed solitons in fibre systems. Such soliton

and their behaviour have been predicted by theories based on complex nonlinear equations, but experimental verification of solitons and their different types has proved challenging.

Prof Tang's search for solitons has focused on using single mode optical fibres. These are specially designed fibres that

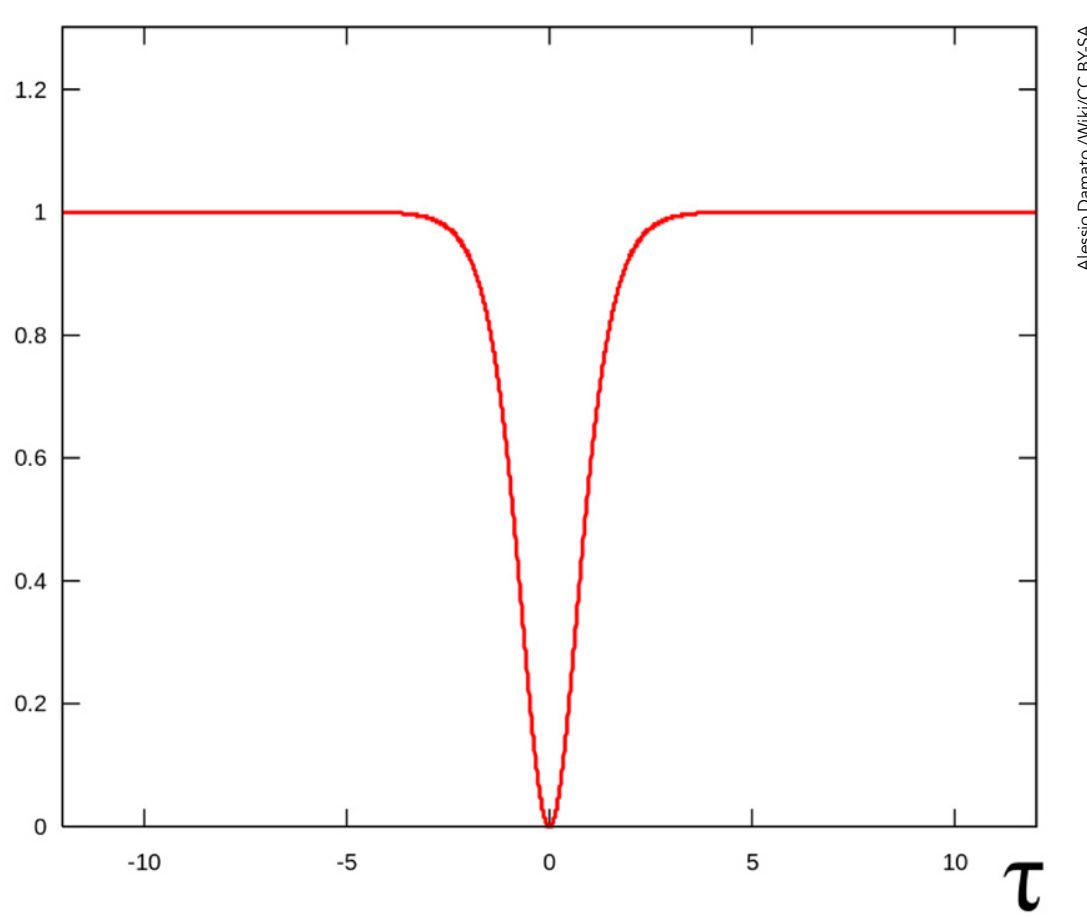




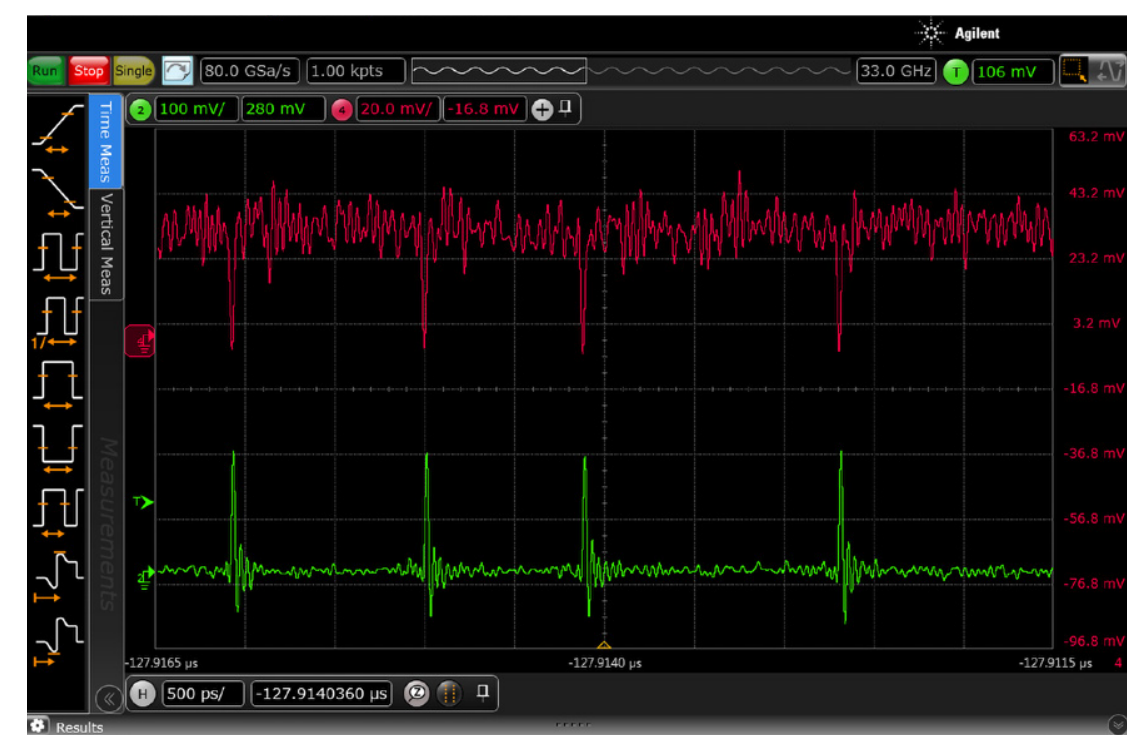

Laser emissions along two orthogonal polarisation directions of light under coherent cross-polarisat
coupling condition. Upper trace: dark soliton enission. Bottom trace: bright soliton emission.

Prof Tang and his team's remarkable achievement is an important step in understanding the accuracy of the nonlinear equations used to model soliton formation.

of light to pass through. The pattern of As well as controlling the mode, the type light depends on how the electric field oscillates relative to its direction of travel and a single light pulse can contain many different modes. Use of single mode fibres restricts this to just one mode of dispersion applied by the fibre can also be tuned. Fibres can be designed to apply either normal, or anomalous dispersion, which affects the shape of the light pulse differenty lead to the formation of different types

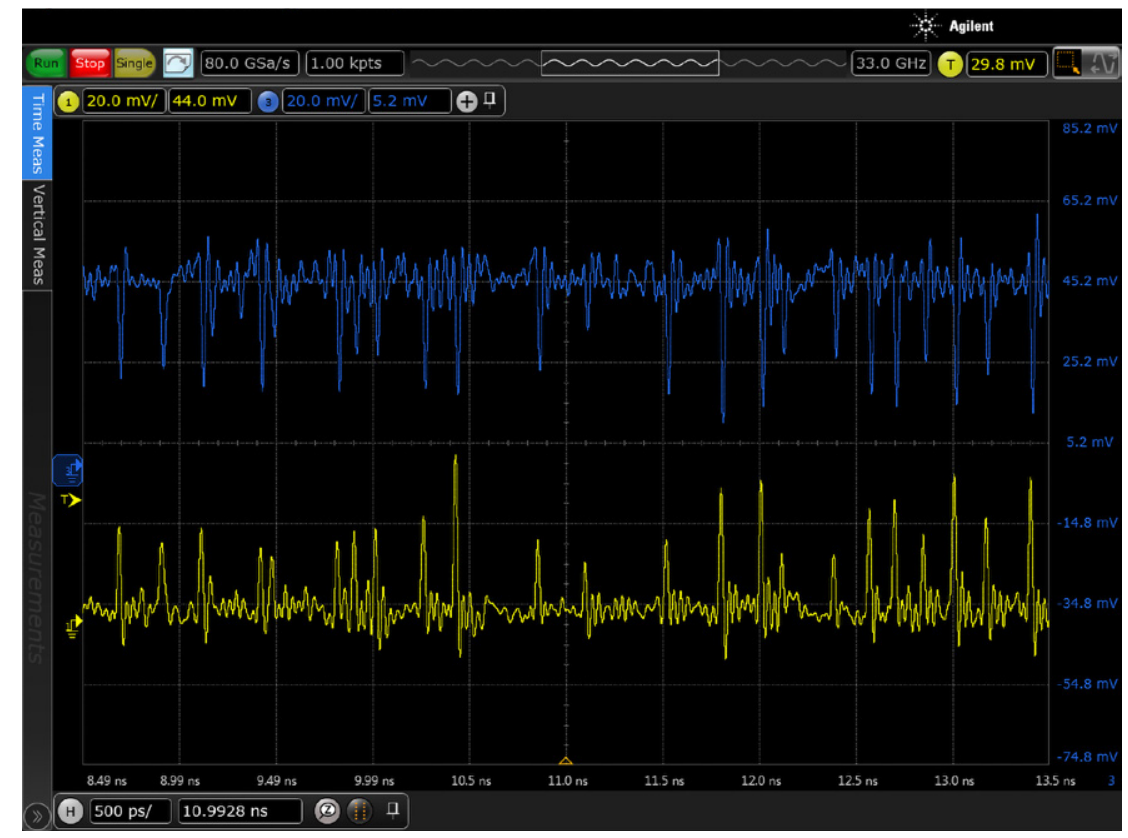
Laser emissions along two orthogonal polarisation directions of light under incoherent cross-pold
coupling condition. Upper trace: dark soliton emission. Bottom trace: bright soliton emission. of solitons. Bright solitons are formed in anomalous dispersion fibres, whereas dispersion materials.

POLARISATION PRECISION

There is one more property of light

that affects the type of solitons formed

transverse waves of the kind produced

by the single mode fibres Prof Tang

used, the polarisation of the light refers

to the relative direction of the oscillating

electric field and direction of travel of

the wave.

As well as dispersion, these fibres also have a small amount of birefringence that induces changes in the polarisation of the light. This allows for the formation solitons, where not just the whole shape of the light pulse maintains its shape on passing through the fibre, but the polarisation components of the light are

To control all of these aspects of the light pulse required Prof Tang and his team to design and build a very specific type of fibre laser that could be used in either the normal or anomalous dispersion regime with very careful control over the amount of birefringence.

y doing this, the team could form either dark-bright or anti-dark-bright vector solitons simultaneously, the first time that they had been observed experimentally after therr theoretical were also able to see that dark-dark (as opposed to single dark solitons) bright-bright solitons could be formed in the birefringent media, suggesting that such coupled solitons were form due to the cross-polarisation of the light fields.

Prof Tang and his team's remarkable achievement is an important step in understanding the accuracy of the nonlinear equations used to model soliton formation. By verifying these predictions, they have shown that these non linear models can be used for subsequent optical fibre design, be it for inre lasers or optical communication opening up new possibilities for the
reaction of photonics technologies.

\section{Behind the Research}

Professor Dingyuan Tang

E: edytang@ntu.edu.sg T: $+6567904337 \quad$ W: https://bit.ly/2zkfv1d

Research Objectives

Professor Tang's research experiments have confirmed the existence of dark-bright and anti-dark-bright vector 18 years ago.

\section{Detail}

Dingyuan Tang

Nenolof Eletrical ar Singapore

\section{Bio}

Prof Tang received his PhD in Physics from University of Hannover, Germany in 1993. His current research is in the fields of laser physics and technology, nonlinear optics, and ceramic laser materials.

Funding National Natural Science Foundation of China (NSFC); Minister of Education (MOE) of Singapore

\section{Collaborators}

-Prof Luming Zhao, Jiangsu Normal University, China - Prof Deyuan Shen, Fudan University, China

\section{NANYANG \\ 10\% UNIVERSITY SINGAPOR}
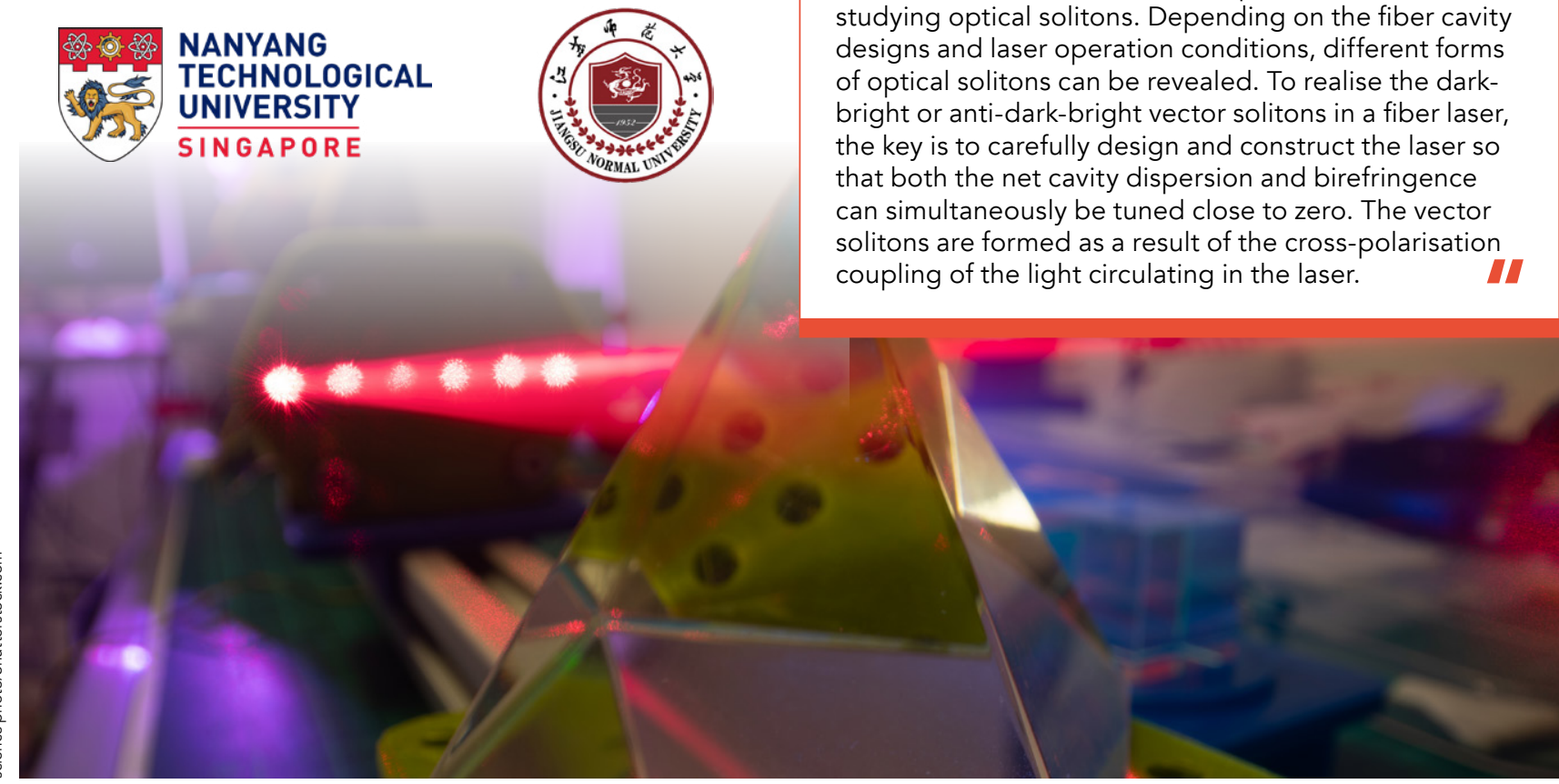

\section{References}

Kivshar, Y.S. (1991). Dark optical solitons near the zerodispersion wavelength. Optics Letters, 16(12), 892. Available at: https://doi.org/10.1364/ol.16.000892

Ma, J., Shao, G.D., Song, Y.F., Zhao, L.M., Xiang, Y.J., Shen, D.Y.,... Tang, D.Y. (2019). Observation of dark-bright vector soll ons in fiber.

Hu, X., Guo, J., Shao, G.D., Song, Y.F., Yoo, S.W., Malomed, B.A., and Tang, D.Y. (2019). Observation of mode fibers. Optics Express, 27(13), 18311. Available at: https://doi.org/10.1364/oe.27.018311

Guo, J., Hu, X., Song, Y.F., Shao, G.D., Zhao, L.M., and Tang, D.Y. (2019). Observation of vector solitons supported Available at: https://doi.org/10.1103/PhysRevA.99.061802

\section{Personal Response}

What was the most challenging part of developing the fibre laser?

II A fiber laser is an excellent experimental testbed for studying optical solitons. Depending on the fiber cavity designs and laser operation conding ons, different forms bright or solti-dark-bight vector solitons in a fiber lascoupling of the light circulating in the laser. 